\title{
Assessment of the EuroSCORE risk scoring system for patients undergoing coronary artery bypass graft surgery in a group of Iranian patients
}

\author{
Hamidreza Jamaati, Arvin Najafi ${ }^{1}$, Farima Kahe ${ }^{1}$, Zahra Karimi ${ }^{1}$, Zarghamhossein Ahmadi, \\ Mohammadreza Bolursaz ${ }^{2}$, Mohammadreza Masjedi, Aliakbar Velayati², \\ Seied Mohammadreza Hashemian
}

Background and Aims: Previous studies around the world indicated validity and accuracy of European System for Cardiac Operative Risk Evaluation (EuroSCORE) risk scoring system we evaluated the EuroSCORE risk scoring system for patients undergoing coronary artery bypass graft (CABG) surgery in a group of Iranian patients. Materials and Methods: In this cohort 2220 patients more than 18 years, who were performed CABG surgery in Massih Daneshvari Hospital, from January 2004 to March 2010 were recruited. Predicted mortality risk scores were calculated using logistic EuroSCORE and Acute Physiology and Chronic Health Evaluation II (APACHE II) and compared with observed mortality. Calibration was measured by the Hosmer-Lemeshow (HL) test and discrimination by using the receiver operating characteristic (ROC) curve area. Results: Of the 2220 patients, in hospital deaths occurred in 270 patients (mortality rate of $12.2 \%$ ). The accuracy of mortality prediction in the logistic EuroSCORE and APACHE II model was 89.1\%; in the local EuroSCORE (logistic) was $91.89 \%$; and in the local EuroSCORE support vector machines (SVM) was $98.6 \%$. The area under curve for ROC curve, was 0.724 (95\% confidence interval [Cl]:0.57-0.88) for logistic EuroSCORE; 0.836 (95\% Cl:0.73 I-0.942) for local EuroSCORE (logistic); 0.978 (95\% Cl: 0.937-I) for Local EuroSCORE (SVM); and 0.832 (95\% Cl: 0.723-0.94I) for APACHE II model.The HL test showed good calibration for the local EuroSCORE (SVM), APACHE II model and local EuroSCORE (logistic) $(P=0.823, P=0.748$ and $P=0.06$ respectively); but there was a significant difference between expected and observed mortality according to EuroSCORE model $(P=0.033)$. Conclusion: We detected logistic EuroSCORE risk model is not applicable on Iranian patients undergoing CABG surgery.

Keywords: Coronary artery bypass graft, European System for Cardiac Operative Risk Evaluation, mortality, risk stratification, scoring system, validity

\section{Introduction}

In cardiac surgery the precise estimate models of risks is most important for surgeons and patients. ${ }^{[1]}$

\section{From:}

Chronic Respiratory Disease Research Center, ${ }^{2}$ Pediatric Respiratory Disease Research Center, National Research Institute of Tuberculosis and Lung Diseases, Shahid Beheshti University of Medical Sciences, Tehran,

${ }^{1}$ Tehran University of Medical Sciences, Tehran, Iran

\section{Correspondence:}

Dr. Seied Mohammadreza Hashemian, Chronic Respiratory Disease Research Center, National Research Institute of Tuberculosis and Lung Diseases, Shahid Beheshti University of Medical Sciences, Tehran, Iran. E-mail: parsmedicine@gmail.com

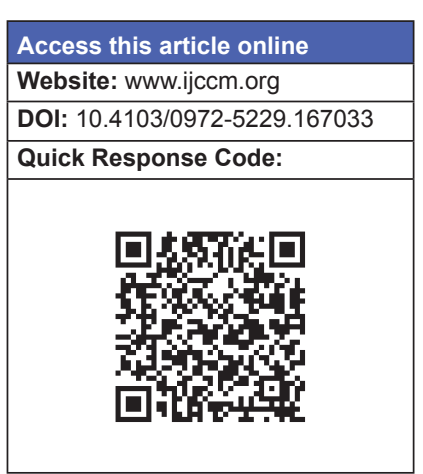

This technique provides a useful tool for surgeons to make a correct decision whether coronary artery bypass graft (CABG) is a suitable intervention, which patients

This is an open access article distributed under the terms of the Creative Commons Attribution-NonCommercial-ShareAlike 3.0 License, which allows others to remix, tweak, and build upon the work non-commercially, as long as the author is credited and the new creations are licensed under the identical terms.

For reprints contact: reprints@ @medknow.com

How to cite this article: Jamaati H, Najafi A, Kahe F, Karimi Z, Ahmadi Z, Bolursaz M, Masjedi M, Velayati A, Hashemian SM. Assessment of the EuroSCORE risk scoring system for patients undergoing coronary artery bypass graft surgery in a group of Iranian patients. Indian J Crit Care Med 2015;19:576-9. 
should be carefully managed and monitored due to adverse outcomes of the operation; ${ }^{[2]}$ furthermore the surgeons can properly inform patients and take preoperative consent. ${ }^{[2]}$ Moreover risk models are a helpful tool to detect differences in risk profiles and to organize the maximum use of health care resources. ${ }^{[3,4]}$ To predict the risks of CABG surgery several scoring systems were developed by scientists. ${ }^{[5-8]}$ The Parsonnet system was introduced in the USA ${ }^{[5]}$ the European System for Cardiac Operative Risk Evaluation (EuroSCORE) was established, based on European population data ${ }^{[6]}$ the American College of Cardiology/American Heart Association presented a scoring system for prediction of outcomes after isolated CABG in America ${ }^{[7]}$ and Society of Cardiothoracic Surgeons of Great Britain and Ireland suggested the UK CABG Bayes model for UK patients undergoing CABG. ${ }^{[8]}$ The EuroSCORE is the most prevalent referenced and was established during 1995-1999 to present a risk model in adult European patients under cardiac surgery. ${ }^{[6,9]}$ It was formed to predict operative mortality, the length of stay in the Intensive Care Units (ICU) ${ }^{[10]}$ complications ${ }^{[11,12]}$ and costs in cardiac surgery. ${ }^{[13,14]}$ EuroSCORE risk models consisted of 17 independent factors and evaluate mortality during 30 days after cardiac surgery and it is widely accepted in Europe and elsewhere. ${ }^{[14]}$ This study aimed to assess the EuroSCORE risk scoring system in patients undergoing CABG surgery in a group of Iranian patients.

\section{Materials and Methods}

As an observational, prospective cohort study 2220 patients more than 18 years undergoing CABG surgery who admitted in ICU, from January 2004 to March 2010 were recruited. The study was approved by the Ethical Committee of our university. Moreover the study procedure was explained for all patients and informed written consents were taken. To assess the EuroSCORE and Acute Physiology and Chronic Health Evaluation II (APACHE II) the patients were fully examined before operation moreover demographic data such as age, sex, race, patient risk factors and comorbidities related to short-term and long-term outcomes, patient disposition, and complications of care were extracted from medical records of patients. Duration of ICU and hospital stay and the possibility of death (main end point of the study) in ICU admission $1^{\text {st }}$ day based on logistic EuroSCORE and APACHE II score were assessed. The coefficients of EuroSCORE variables were re-estimated on this group as two local EuroSCORE models, with logistic regression and support vector machines (SVM) and the discriminative power and calibration of these models were compared. In order to assess the EuroSCORE calibration, the Hosmer-Lemeshow (HL) test of goodness of fit was applied. ${ }^{[15]}$ The accuracy of the model was calculated by the receiver operating characteristic (ROC) curve (ROC curve), designed for sensibility (accurate death prediction) and specificity (accurate prediction of survival), analyzed for each value of each score studied. The area under curve (AUC) was defined as the area under the ROC curve. The 0.5 AUC value indicates a random distinguishing of the patients being alive and dead. An increasing value of AUC from 0.5 toward 1.0 shows increasing distinctiveness and better discrimination of the patients' status. AUC values were calculated for logistic EuroSCORE and APACHE II and logistic regression and SVM models to test discrimination and to describe performance and accuracy. Categorical variables are displayed as numbers and/or percentages, and continuous variables are displayed as mean \pm standard deviation. The results were presented with $95 \%$ confidence intervals (CIs). A two-sided $P<0.05$ was considered as statistical significance. The statistical program employed was SPSS (Statistical Package for the Social Sciences) - version 18.0 for windows.

\section{Results}

A total of 2220 patients undergoing cardiac surgery between January 2004 and March 2010 were evaluated. The clinical characteristics of our patients and the EuroSCORE are presented in Table 1. There were significant differences between our patient group and European cardiac surgical populations. In comparison the patients in current survey were younger than the European patients. Moreover, our patients were more prone to have unstable angina, moderate left

\begin{tabular}{lcc}
\hline $\begin{array}{l}\text { Table I: Demonstrates comparative prevalence of risk } \\
\text { factors in Iranian and European population }\end{array}$ \\
\hline Risk factor & $\begin{array}{c}\text { Iranian } \\
\text { prevalence (\%) }\end{array}$ & $\begin{array}{c}\text { EuroSCORE } \\
\text { prevalence (\%) }\end{array}$ \\
\hline$n$ & 740 & 19,030 \\
Mean age (year) & 57 & 62.5 \\
Female & 49 & 27.8 \\
Chronic pulmonary disease & 11.5 & 3.9 \\
Extracardiac arthropathy & 2.7 & 11.3 \\
Neurological disease & 4.1 & 1.4 \\
Previous cardiac surgery & 12.2 & 7.3 \\
Serum creatinine > 200 $\mu$ mol/L & 6.8 & 1.8 \\
Active endocarditis & 2.7 & 1.1 \\
Critical preoperative state & 4.1 & 4.1 \\
Unstable angina & 79.7 & 8 \\
LV dysfunction moderate or LVEF & 56.1 & 25.6 \\
30-50\% & & \\
LV dysfunction poor or LVEF $<30$ & 7.4 & 5.8 \\
Recent myocardial infarct & 6.8 & 9.7 \\
Pulmonary hypertension & 8.1 & 2 \\
Emergency surgery & 12.8 & 4.9 \\
Other than isolated CABG & 33.8 & 36.4 \\
Surgery on thoracic aorta & 1.4 & 2.4 \\
Postinfarct septal rupture & 0.7 & 0.2 \\
\hline LV: Left ventricular; LVEF: Left ventricular ejection fraction; CABG: Coronary artery \\
bypass graft & & \\
& &
\end{tabular}


ventricular function, critical preoperative state, active endocarditis, chronic pulmonary disease compared to European population. Fewer patients have recent myocardial infarction, extra cardiac arteriopathy, and surgery on thoracic aorta. The prevalence of female sex, neurological disease, serum creatinine $>0.2 \mathrm{~mol} / \mathrm{l}$, pulmonary hypertension, emergency surgery, previous cardiac surgery, and surgery other than isolated CABG surgery and postinfarct septal rupture was higher than European population. Of the 2220 patients, in hospital deaths occurred in 270 patients (mortality rate of $12.2 \%$ ). The accuracy of mortality prediction in the logistic EuroSCORE and APACHE II model was 89.1\%; in the local EuroSCORE (logistic) was $91.89 \%$; and in the local EuroSCORE (SVM) was $98.6 \%$. The AUC for ROC (ROCs) curve, was 0.724 (95\% CI: 0.57-0.88) for logistic EuroSCORE; 0.836 (95\% CI: 0.731-0.942) for local EuroSCORE (logistic); 0.978 (95\% CI: 0.937-1) for local EuroSCORE (SVM); and 0.832 (95\% CI: 0.723-0.941) for APACHE II model [Figure 1]. The HL goodness of fit test showed good calibration for the local EuroSCORE (SVM), APACHE II model and local EuroSCORE (logistic) $(P=0.823, P=0.748$ and $P=0.06$ respectively); but there was a significant difference between expected and observed mortality according to EuroSCORE model $(P=0.033)$. In order to models calibration HL test was applied [Figure 2].

\section{Discussion}

It is well-established in some studies that EuroSCORE is discriminative and accurate model predictions of operative mortality, ${ }^{[16]}$ however, previous study in Iran revealed it is not valid in Iranian population. ${ }^{[17]}$ The aim of this experience was to evaluate the EuroSCORE in CABG surgery in Iranian patients. Therefore we evaluated the validity of the logistic EuroSCORE model

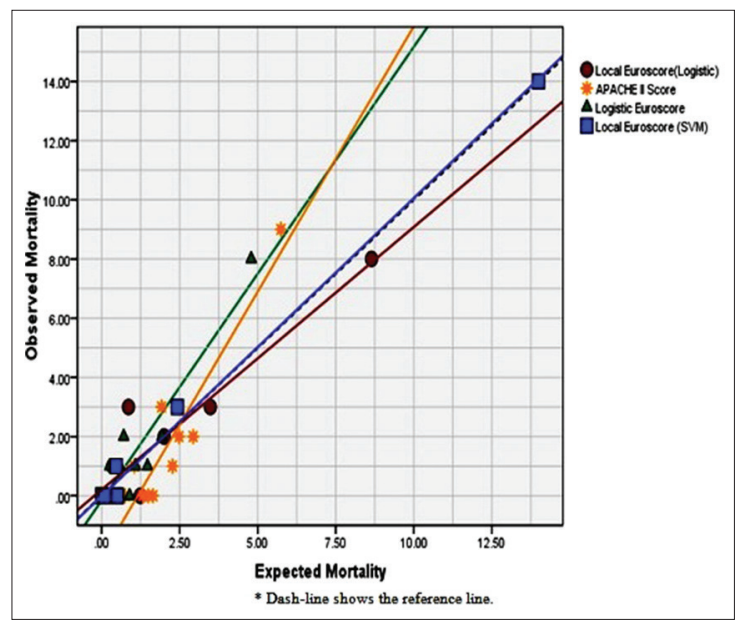

Figure I: The calibration plot for comparing four different models in Iranian patients undergoing cardiac surgery by testing its calibration power and discrimination power. Discrimination power was assessed by calculating area under ROC curve which was 0.724 (95\% CI: 0.57-0.88) for logistic EuroSCORE. The discriminatory power was considered excellent if AUC was $>0.80$, very good if $>0.75$, and good if $>0.70 .^{[18]}$ In summary the results of our survey indicated that logistic EuroSCORE risk model is not accurate for predicting mortality at all risk subgroups in Iranian patients. Moreover we detected considerable differences in patient demographics between the Iranian and 1995 EuroSCORE data sets. In line with our experience Yap et al. reported that the model was not valid in Australia because of different patient characteristics and different prevalence of risk factors. They revealed a significant low mortality in contrast to predicted mortality by means of EuroSCORE data sets. ${ }^{[19]}$ Harmoniously Bhatti et al. prospectively evaluated British data containing 9995 patients from "North West Quality Improvement Program in Cardiac Interventions." They reported that the discrimination power of the logistic EuroSCORE was good with ROC curve area of 0.79 for all types of cardiac surgery, but overestimated in-hospital mortality. ${ }^{[20]}$ In accordance with Bhatti et al. study D'Errigo et al. observed 30,610 isolated CABG surgeries and found that their observed mortality was significantly lower than the predicted mortality according to the logistic EuroSCORE. ${ }^{[2]}$ These studies were not surprising because the EuroSCORE scoring system was introduced 15 years ago. Because to date, there have been wonderful advancements in surgical methods, anesthetic and postoperative intensive care quality. Consequently, all of these have led to better surgical results and reduced mortality. On the other hand, although the EuroSCORE database is UpToDate and several studies indicated that, the EuroSCORE model has been validated on patients

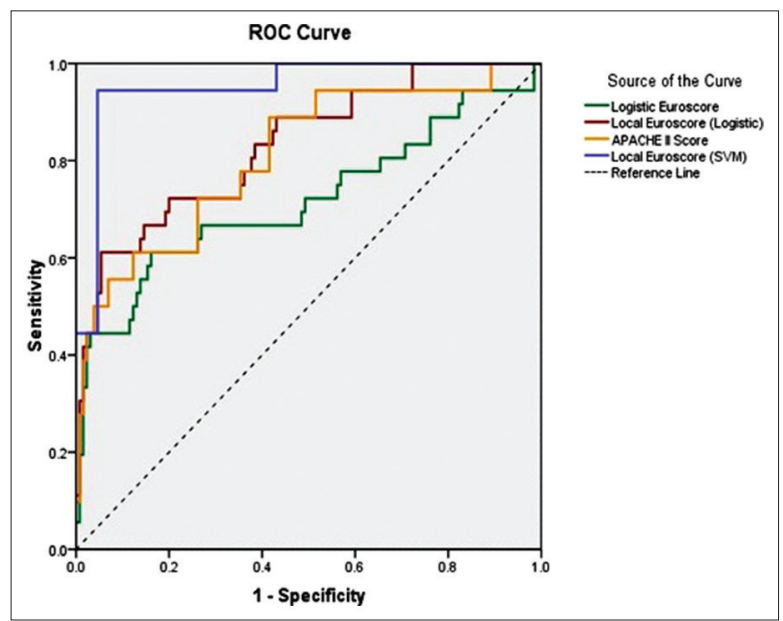

Figure 2: The receiver operating characteristic curve for comparison of discriminative power of different models 
in Japan ${ }^{[22]}$ and North American. ${ }^{[23]}$ However, it is derived from a cross-section of contemporary European cardiac surgery. So, it may appropriate database for the construction of a risk evaluation scoring system for use in Europe and it may provide conflicting results in CABG patients in other regions like Iran (West of Asia). In line with us a study in Thailand in Southeast of Asia showed EuroSCORE is not valid ${ }^{[2]}$ and in Netherland in north of Europe van Straten et al. indicated additive and logistic Euroscore are overestimating mortality rate. ${ }^{[25]}$ Additionally, important developments in CABG surgery methods and postoperative care after the creation of the risk scoring systems also should be considered.

\section{Conclusion}

We detected logistic EuroSCORE risk model is not applicable on Iranian patients' undergoing CABG surgery. However, larger studies are required to confirm results reported here. Moreover due to demographic difference between Iranian and European patients, creation a new specific and local risk stratification system is essential for new experiences in Iranian patients.

\section{Financial support and sponsorship \\ Nil.}

\section{Conflicts of interest}

There are no conflicts of interest.

\section{References}

1. Ferraris VA, Ferraris SP. Risk stratification and comorbidity. In: Cohn LH, Edmunds LH Jr, editors. Cardiac Surgery in the Adult. $2^{\text {nd }}$ ed. New York: McGraw-Hill; 2003. p. 187-224.

2. Hammermeister KE. Risk, predicting outcomes, and improving care. Circulation 1995;91:899-900.

3. Nashef SA, Roques F, Michel P, Cortina J, Faichney A, Gams E, et al. Coronary surgery in Europe: Comparison of the national subsets of the European system for cardiac operative risk evaluation database. Eur J Cardiothorac Surg 2000;17:396-9.

4. Smith PK, Smith LR, Muhlbaier LH. Risk stratification for adverse economic outcomes in cardiac surgery. Ann Thorac Surg 1997;64 6 Suppl: S61-3.

5. Parsonnet V, Dean D, Bernstein AD. A method of uniform stratification of risk for evaluating the results of surgery in acquired adult heart disease. Circulation 1989;79 (6 Pt 2):I3-12

6. Nashef SA, Roques F, Michel P, Gauducheau E, Lemeshow S, Salamon R. European system for cardiac operative risk evaluation (EuroSCORE). Eur J Cardiothorac Surg 1999;16:9-13.

7. Eagle KA, Guyton RA, Davidoff R, Ewy GA, Fonger J, Gardner TJ, et al. ACC/AHA guidelines for coronary artery bypass graft surgery: Executive summary and recommendations: A report of the American College of Cardiology/American Heart Association Task Force on Practice Guidelines (Committee to revise the 1991 guidelines for coronary artery bypass graft surgery). Circulation 1999;100:1464-80.
8. Anonymous. The Society of Cardiothoracic Surgeons of Great Britain and Ireland. National Adult Cardiac Surgical Database Report. Final draft. London: SCTS; 1998.

9. Roques F, Nashef SA, Michel P, Gauducheau E, de Vincentiis C, Baudet E, et al. Risk factors and outcome in European cardiac surgery: Analysis of the EuroSCORE multinational database of 19030 patients. Eur J Cardiothorac Surg 1999;15:816-22.

10. Nilsson J, Algotsson L, Höglund P, Lührs C, Brandt J. EuroSCORE predicts intensive care unit stay and costs of open heart surgery. Ann Thorac Surg 2004;78:1528-34.

11. Toumpoulis IK, Anagnostopoulos CE, Swistel DG, DeRose JJ Jr. Does EuroSCORE predict length of stay and specific postoperative complications after cardiac surgery? Eur J Cardiothorac Surg $2005 ; 27: 128-33$.

12. Toumpoulis IK, Anagnostopoulos CE, DeRose JJ, Swistel DG. Does EuroSCORE predict length of stay and specific postoperative complications after coronary artery bypass grafting? Int J Cardiol 2005; 105:19-25.

13. Pinna Pintor P, Bobbio M, Colangelo S, Veglia F, Marras R, Diena M. Can EuroSCORE predict direct costs of cardiac surgery? Eur J Cardiothorac Surg 2003;23:595-8.

14. Australian Society of Cardiac and Thoracic Surgeons Cardiac Surgery Database Project-Surgeon Report; 2004.

15. Collart F, Feier H, Kerbaul F, Mouly-Bandini A, Riberi A, Mesana TG, et al. Valvular surgery in octogenarians: Operative risks factors, evaluation of Euroscore and long term results. Eur J Cardiothorac Surg 2005;27:276-80.

16. Guida P, Mastro F, Scrascia G, Whitlock R, Paparella D. Performance of the European System for Cardiac Operative Risk Evaluation II: A meta-analysis of 22 studies involving 145,592 cardiac surgery procedures. J Thorac Cardiovasc Surg 2014;148:3049-57.e1.

17. Sadeghi MM, Arasteh M, Gharipour M, Nilfroush P, Shamsolketabi H, Etesampour A, et al. Evaluation of accuracy of Euroscore risk model in prediction of perioperative mortality after coronary bypass graft surgery in Isfahan. J Res Med Sci 2011;16:787-92.

18. Lovegrove J, Valencia O, Treasure T, Sherlaw-Johnson C, Gallivan S. Monitoring the results of cardiac surgery by variable life-adjusted display. Lancet 1997;350:1128-30.

19. Yap CH, Reid C, Yii M, Rowland MA, Mohajeri M, Skillington PD, et al. Validation of the EuroSCORE model in Australia. Eur J Cardiothorac Surg 2006;29:441-6.

20. Bhatti F, Grayson AD, Grotte G, Fabri BM, Au J, Jones M, et al. The logistic EuroSCORE in cardiac surgery: How well does it predict operative risk? Heart 2006;92:1817-20.

21. D'Errigo P, Seceareceia F, Rosato S, Manno V, Badoni G, Fusco D, et al. Comparison between an empirically derived model and the EuroSCORE system in the evaluation of hospital performance: The example of the Italian CABG Outcome Project. Eur J Cardiothorac Surg 2008;33:325-33.

22. Kawachi Y, Nakashima A, Toshima Y, Arinaga K, Kawano H. Risk stratification analysis of operative mortality in heart and thoracic aorta surgery: Comparison between Parsonnet and EuroSCORE additive model. Eur J Cardiothorac Surg 2001;20:961-6.

23. Nashef SA, Roques F, Hammill BG, Peterson ED, Michel P, Grover FL, et al. Validation of European System for Cardiac Operative Risk Evaluation (EuroSCORE) in North American cardiac surgery. Eur J Cardiothorac Surg 2002;22:101-5.

24. Karunasumetta C, Laksanabunsong P, Slisatkorn W, Wongkornrat W, Sakiyalak P, Thongcharoen P, et al. Validation of EuroSCORE for coronary artery bypass grafting at Siriraj Hospital. J Med Assoc Thai 2012:95:1178-83.

25. van Straten AH, Tan EM, Hamad MA, Martens EJ, van Zundert AA Evaluation of the EuroSCORE risk scoring model for patients undergoing coronary artery bypass graft surgery: A word of caution. Neth Heart J 2010;18:355-9. 